\title{
Tsafon
}

Revue d'études juives du Nord

$81 \mid 2021$

Des synagogues à travers les âges Lieux de prières, lieux d'études et autres fonctions

\section{Circulaire de René Bousquet, relative aux « Agissements des Juifs » (1943)}

\section{Monique Heddebaut}

\section{(2) OpenEdition \\ Journals}

Édition électronique

URL : https://journals.openedition.org/tsafon/3963

DOI : $10.4000 /$ tsafon.3963

ISSN : 2609-6420

Éditeur

Association Jean-Marie Delmaire

Édition imprimée

Date de publication : 1 juillet 2021

Pagination : 133-143

ISSN : $1149-6630$

Référence électronique

Monique Heddebaut, "Circulaire de René Bousquet, relative aux « Agissements des Juifs » (1943)», Tsafon [En ligne], 81 | 2021, mis en ligne le 01 juillet 2021, consulté le 16 septembre 2021. URL : http:// journals.openedition.org/tsafon/3963; DOI : https://doi.org/10.4000/tsafon.3963 


\author{
Édition : Histoire
}

\title{
Circulaire de René Bousquet,
}

\section{relative aux « Agissements des Juifs » (1943)}

\author{
Monique Heddebaut ${ }^{*}$
}

Le document reproduit ci-après (voir page suivante) est une circulaire conservée aux Archives départementales du Pas-de-Calais, classée dans les archives modernes, elles-mêmes divisées en quatorze séries, qui s'étendent de 1800 à 1975. Les six sous-préfectures du Pas-deCalais existantes avant $1962^{1}$ ont versé des documents classés dans la sous-série 1 à $6 \mathrm{Z}$ et qui étaient mêlés autrefois à ceux de la préfecture dans les différentes séries du cadre réglementaire.

Cette circulaire cotée 1 Z 497 en date du 20 août 1943 provient de la sous-préfecture de Béthune. Elle appartient à un dossier intitulé : « Organisation des immeubles appartenant à des israélites ». Elle a pour objet l'" Agissement des Juifs ». Elle n'a pas été identifiée dans d'autres fonds d'archives du Nord-Pas-de-Calais. Il s'agit d'une seule feuille, sur un recto, et d'un texte composé de sept paragraphes de quelques lignes, soit 20 lignes au total.

\footnotetext{
${ }^{*}$ Commission historique du Nord et Centre de recherche sur la barbarie et la déshumanisation (CRIBED), Lille.

${ }^{1}$ Les sous-préfectures de Calais et de Lens ont été créées en 1962 par démembrement de celles de Boulogne-sur-Mer et de Béthune.
} 


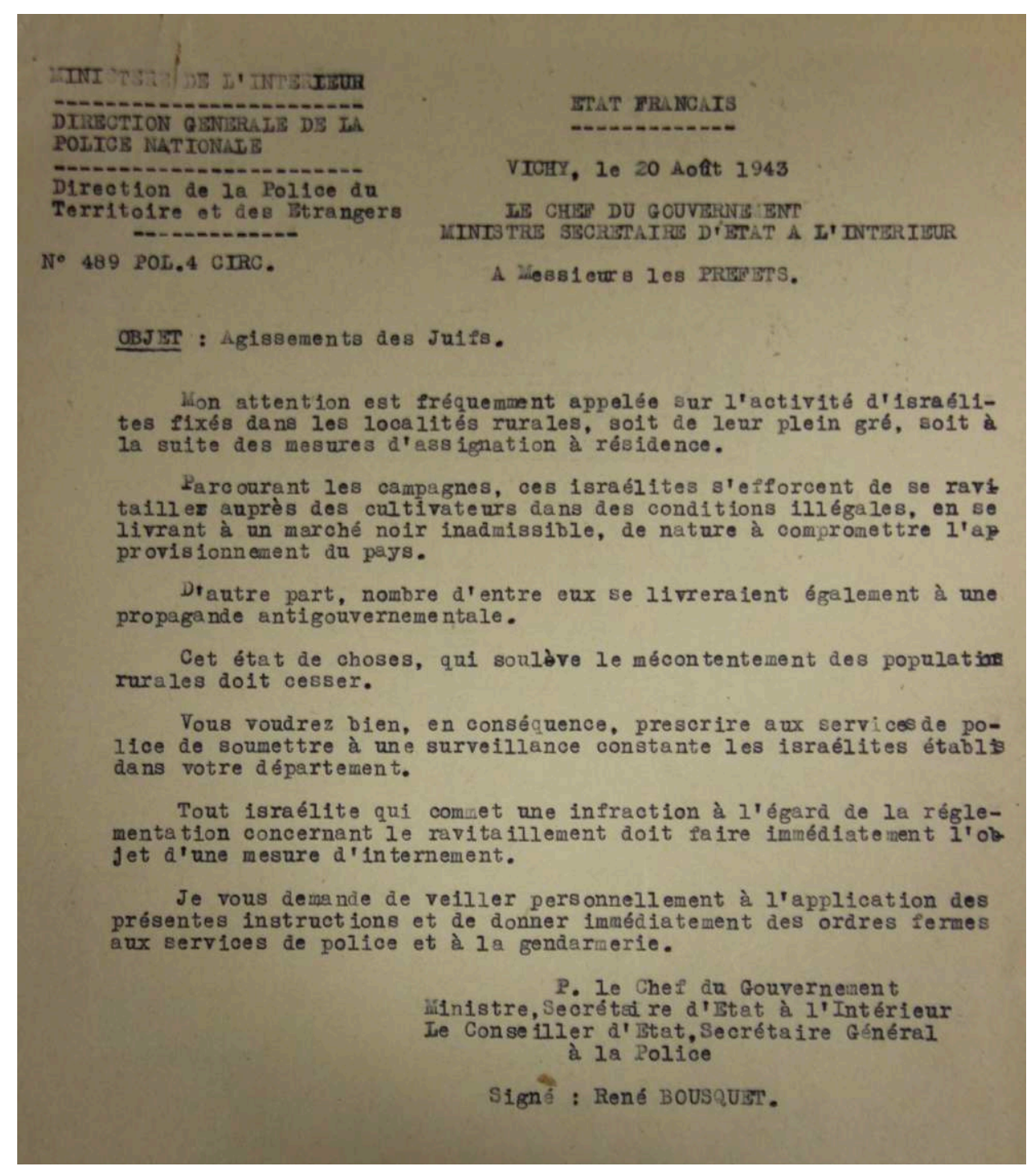

Circulaire en date du 20 août 1943 intitulée : « Organisation des immeubles appartenant à des israélites » objet : « Agissement des Juifs ».

Archives départementales du Pas-de-Calais : 1 Z 497 


\section{L'auteur et les destinataires}

\section{René Bousquet, secrétaire général de la Police}

Cette circulaire du 20 août 1943 émane de l'État français installé à Vichy depuis que la Troisième République a été remplacée par un nouveau régime, faisant ainsi disparaître la mention «République française » des actes officiels. René Bousquet la signe en tant que secrétaire d'État à l'Intérieur, conseiller d'État et secrétaire général à la Police, pour le chef du gouvernement et ministère de l'Intérieur, Pierre Laval.

Cet homme aux importantes responsabilités est né le 11 mai 1909 à Montauban (Tarn-et-Garonne). Fils de notaire, radical-socialiste, il est devenu chef de cabinet du préfet du Tarn-et-Garonne à vingt ans. Il a continué sa carrière et son ascension fulgurante en intégrant le gouvernement de Vichy : il a été nommé secrétaire général de la Police le 18 avril 1942 dans le ministère de l'Intérieur nouvellement dirigé par Pierre Laval. Il est resté en poste jusqu'au 31 décembre 1943. Il s'est employé à faire reconnaître la pleine souveraineté de Vichy sur l'ensemble du territoire français par les Allemands, y compris dans la zone occupée. Une plainte a été déposée contre lui en 1989 pour crime contre l'humanité. Mais il a été abattu le 8 juin 1993 à Paris par Christian Didier éteignant ainsi l'affaire dont l'instruction était en cours ${ }^{2}$.

Bousquet est étroitement associé à l'organisation des arrestations des Juifs étrangers dans les zones nord et sud. Il a négocié les 2 et 3 juillet 1942, leur déportation avec Karl Oberg, chef suprême de la SS et de la police allemande en France. Ce marchandage est connu sous le nom d'accords « Bousquet-Oberg » qui ont officialisé la coopération française à la déportation. Il a accepté que la police française effectue une rafle qui prévoyait l'arrestation de 22000 Juifs, lors de la rafle dite du Vel' d'Hiv' les 16 et 17 juillet 1942 .

Il en avait exposé ainsi l'organisation deux semaines auparavant, le 6 juillet 1942, lors d'une conférence des préfets régionaux à Paris présidée par Laval :

Le gouvernement allemand a demandé de faire arrêter par la police française 40000 Juifs dont 20000 Français en zone occupée pour déportation, plus de 10000 en zone libre.

\footnotetext{
${ }^{2}$ Renseignements biographiques tirés de Jean-Paul Cointet, Les hommes de Vichy, L'Illusion du pouvoir, Paris, Perrin, 2017, 374 p.
} 
Juifs apatrides ou étrangers

Possibilité de lui donner satisfaction. On les conduira dans un territoire de l'Est. $\underline{\text { Juifs français }}$

Le gouvernement ne veut pas faire de politique trop antisémite. Il faut créer un statut national pour les Juifs français. Il n'est plus question des Juifs français pour la déportation $[\ldots] .^{3}$

Ce sont finalement 13152 Juifs qui ont été remis aux autorités d'occupation en vue d'une déportation à l'Est ${ }^{4}$. Les relations entre le gouvernement de Vichy, Bousquet et les autorités allemandes vont ensuite se cristalliser pendant l'année 1943 autour d'un accord problématique à trouver sur la déportation des Juifs français, protégés par leur nationalité, les Juifs étrangers restant les cibles toutes désignées.

\section{Fernand Carles, préfet régional du Nord}

Le préfet régional du Nord chargé d'appliquer la circulaire du 20 août 1943 était Fernand Carles, haut fonctionnaire qui fut en poste à Lille à partir de 1936 jusqu'au 2 septembre 1944, date à laquelle il fut arrêté lors de la libération de la ville. Il est mort en détention le 23 avril 1945 dans des conditions qui n'ont pu être éclaircies. Il était secondé par le préfet délégué Henry Darrouy, de 1941 à 1944, et le préfet du Pas-de-Calais, André Sadon, d'avril 1943 à janvier 1944.

Dans quelle mesure les autorités préfectorales d'une région qui a relevé du Commandement militaire pour la Belgique et le Nord de la France [Militärbefehlshaber für Belgien und Norfrankreich] dès la mi-juin 1940, ont-elles pu, voulu et souhaité appliquer les directives venues du gouvernement de Vichy et de son secrétaire général de la Police, René Bousquet?

Le général Niehoff, chef de l'OberFeldKommandantur, l'OFK 670, avait avisé les préfets que lui seul détenait la puissance souveraine car disposant du pouvoir législatif, exécutif et judiciaire. Au début de l'installation de l'occupant allemand dans le nord de la France, il a ignoré jusqu'à l'existence même de l'État français, considérant parfois certaines de ses décisions comme nulles et non avenues à moins qu'elles n'aient été validées par lui ${ }^{5}$. En revanche, il a existé très vite un terrain d'entente entre

\footnotetext{
3 Archives départementales du Nord, désormais AD59 1W117, Rapport du préfet Fernand Carles, juillet 1942.

${ }^{4}$ Laurent Joly, L'Etat contre les Juifs, Paris, Grasset, 2018, p. 89.

${ }^{5}$ Étienne Dejonghe, Yves Le Maner, Le Nord-Pas-de-Calais dans la main allemande1940-1944, Lille, La Voix éditions, p. 89.
} 
autorités allemandes et françaises dans le Nord : la chasse aux Juifs et aux communistes.

Fernand Carles était présent à la conférence des préfets régionaux du 6 juillet 1942 où René Bousquet les a informés du nombre de Juifs à déporter. De retour dans le Nord, il a rédigé un rapport destiné à l' « information personnelle » d'Henri Darrouy, son préfet délégué6. Une note de renseignements émanant des services allemands sur la préfecture du Nord et postérieure à novembre 1942 éclaire sur le rôle du préfet Carles. On peut y lire que : « Le cabinet du Préfet joue un rôle très important dans la vie administrative et politique du Département. Il traite [...] de la coordination des Affaires juives $»^{7}$.

Et dans le cas présent, c'est-à-dire la surveillance des « agissements des Juifs » en 1943 réclamée par Bousquet, le préfet Carles, comme tous les préfets du territoire, a appliqué cette directive en toute connaissance de cause, renforçant et parachevant le processus des arrestations et des déportations de l'été 1942. Il est toutefois difficile et malaisé d'appréhender jusqu'à quel point il était devenu un supplétif des autorités d'occupation, de saisir jusqu'où il a joué un double jeu face aux Allemands et à leurs exigences à l'encontre des Juifs étrangers et français. En effet, cet homme de confession protestante avait épousé en 1913 Thérèse Lang, issue d'une lignée de négociants juifs alsaciens. Bien que protégée par sa nationalité française et le poste de son mari, elle a été la cible des antisémites. Utilisant relations et informations, elle joua un rôle important dans le Comité clandestin de secours d'aide aux juifs créé au lendemain de la rafle du 11 septembre1942.

\section{La date et l'objet de la circulaire}

Cette circulaire d'août 1943 est à considérer à l'aune des bilans que les Allemands, aidés par le gouvernement de Vichy, ont dressés après les rafles de l'été 1942 en France et celle du 11 septembre 1942 dans le NordPas-de-Calais. Ils n'ont pas atteint leurs quotas d'arrestations et de déportations qui se sont révélés très en deçà de leurs prévisions. Heinz Röthke, Judenreferat, chef suprême des SS et de la police en France occupée et dirigeant du camp de Drancy de juillet 1942 à juillet 1943, avait calculé qu'au 6 mars 1943, 49000 Juifs avaient été déportés, envoyés à

\footnotetext{
${ }^{6}$ AD59 1W117, Rapport du préfet - Juillet 1942.

${ }^{7}$ AD59 9W511-23, Opinion des Allemands sur les fonctionnaires de la Préfecture et de la police.
} 
l'est, selon sa formule, mais qu'il en restait 270 000, dont 200000 dans la zone $\operatorname{sud}^{8}$. Ces chiffres rejoignent les estimations d'une communauté évaluée en métropole entre 300000 à 330000 personnes $^{9}$. Cette forme d'aveu d'échec partiel va entraîner une riposte et d'autres mesures.

Le ton est donné dès le premier mot de la circulaire qui a pour objet les « agissements des Juifs ». Bousquet désigne précisément par ce terme la façon d'agir des Juifs, qu'il considère comme souvent blâmable, tout comme leurs manœuvres pour arriver à leur but. Pour interpeller les préfets, il s'est réfugié derrière une expression couramment usitée et suffisamment vague pour ne pas avoir à l'étayer, qui fait référence à l'état d'esprit de la population, celle des modérés, des bien-pensants, des tenants de l'ordre établi : «Mon attention est fréquemment appelée », ce qui est invérifiable, mais qui peut impressionner, troubler, poser question. Et il a justifié la prise en compte de l'opinion par l'adverbe « fréquemment », ce qui explique qu'il ne pouvait rester sans réponse face à de tels désordres et qu'il se devait de prendre des mesures afin de maintenir l'ordre public.

Il a utilisé d'abord et une seule fois le terme «Juifs », puis celui d' « Israélites » à quatre reprises dans le corps du texte. Il les a employés comme synonymes, les mêlant sciemment, tout en ne définissant pas le terme « israélite » qui n'a pas de définition juridique, qui ne figurait d'ailleurs ni dans la loi du 3 octobre 1940 portant statut des Juifs, ni dans celle du 2 juin 1941 qui l'a remplacée. Par un glissement sémantique, il a amalgamé les Israélites souvent perçus comme des Français de confession israélite et les Juifs étrangers. Bousquet, haut fonctionnaire chargé de l'application des lois depuis son entrée dans le corps préfectoral en 1929, ne pouvait en méconnaître le sens particulier pendant l'entre-deux guerres où les questions de nationalité ont animé le débat politique.

\section{Bousquet et le monde rural}

D'emblée il est question d'Israélites «fixés dans les localités rurales », ce qui jette le doute sur leur ancrage réel et ancien dans un territoire, dans un terroir. Effectivement, certains de ces Juifs avaient fui

\footnotetext{
${ }^{8}$ Chiffres cités par Michaël R. Marrus et Robert O. Paxton, Vichy et les juifs, Paris, Calmann-Levy, 2015 ( $2^{\mathrm{e}}$ édition), p. 433-434, qui s'appuient sur la note de Röthke du 6 mars 1943, CDJC (Centre de Documentation Juive contemporaine à Paris) : XXVc-214. ${ }^{9}$ André Kaspi, dans Les Juifs pendant l'Occupation, Paris, Le Seuil, coll. «Points Histoire », 1997, p. 2, avance le chiffre de 300000 personnes tout en soulignant les difficultés pour définir le Juif dont la définition juridique n'existe pas en droit français, au moins jusqu'en 1940.
} 
la Belgique et le nord de la France lors de l'invasion allemande en maijuin 1940 ou avaient été assignés à résidence, étape précédant parfois l'internement et la déportation. Rappelons que la France de 1939 était un pays encore fortement rural, que la moitié de la population, soit 20 millions de personnes, vivait dans les campagnes. L'agriculture était un secteur-clé de l'économie du pays. Et la paysannerie avait largement été mise à l'honneur par une intense propagande du régime de Vichy qui jouait les ruraux contre les urbains. Le maréchal Pétain n'avait-il pas déclaré le 25 juin 1940, lorsqu'il a annoncé aux Français les conditions de l'armistice, que : « La terre, elle, ne ment pas. Elle demeure votre recours. Elle est la patrie elle-même ». Il voyait là un des piliers de la France nouvelle dans un monde rural qui serait majoritairement conservateur, catholique et par là même antisémite. Les ruraux se seraient mécaniquement rangés derrière le maréchal Pétain et sa politique de collaboration ${ }^{10}$. Des historiens se sont attachés à déconstruire ce mythe que la propagande du régime de Vichy avait tenté d'accréditer et de répandre. De nombreux témoignages, à l'échelon national et nordiste, montrent que les solidarités et les aides, voire les complicités, ont existé dans le monde paysan, notamment pour les enfants cachés.

Bousquet qui entendait établir que les agissements des Juifs étaient « de nature à compromettre l'approvisionnement du pays », s'est appuyé sur les stéréotypes véhiculés depuis des siècles : le Juif cupide, pilleur, envieux et qui ne serait pas loyal envers le pays d'accueil, car sans attaches, sans patrie. Le chef de la Police visait le monde paysan qui a pu être sensible à la propagande maréchaliste dans les premiers mois du régime, mais qui a manifesté une désaffection croissante, à partir du second semestre 1942, ainsi que l'ont noté certains préfets dans leurs rapports mensuels au gouvernement de Vichy. Et il a ensuite mis en concurrence les Juifs et les non-Juifs. Il a sciemment inversé les rôles à une époque où la France était mise en coupe réglée par l'occupant sous la forme d'un pillage généralisé des ressources industrielles et agricoles qui était organisé au nom de la convention d'armistice. Or, les quelque 270000 Juifs qui restaient à arrêter et à déporter à l'est selon les estimations de Röthke, vivaient soit dans la clandestinité, soit dans une semi-légalité. Chiffre qui représentait entre $0,5 \%$ et $1 \%$ de la population française. Comment auraient-ils pu désorganiser et compromettre l'approvisionnement d'un pays de 40 millions d'habitants?

${ }^{10}$ Ce fut le thème du Concours National de la Résistance et de la Déportation (CNRD) : « Résistance et monde rural » de 2005-2006. 
Cette circulaire met l'accent sur leurs difficultés matérielles et éclaire d'un jour cru et cruel le quotidien de ceux qui avaient réussi à échapper aux rafles, qui vivaient désormais dans la clandestinité et essayaient d'organiser leur subsistance, leur survie, ce dont était parfaitement conscient le secrétaire général à la Police qui cherchait ni plus, ni moins, à les affamer et les identifier de cette façon. Afin d'organiser le ravitaillement général, le rationnement avait été mis en place par décret du 10 mars 1940 par le biais des cartes d'alimentation individuelles et nominatives délivrées par les mairies avec des tickets pour tous les biens de consommation. Cela nécessitait une déclaration et un recensement des personnes alors classées en huit catégories. C'était une difficulté supplémentaire et quasi-insurmontable pour ceux qui s'efforçaient de se rendre invisibles, pour les clandestins, pour ceux qui vivaient sous le coup du statut des Juifs, des interdictions professionnelles, des mesures d'exclusion, pour ceux qui étaient interdits de contacts avec le public et porteurs de l'étoile juive. Ils devaient prendre davantage de risques, à moins de bénéficier de complicités dans la population et parmi les employés de mairie. Ils en étaient réduits à une pauvreté extrême.

Bon nombre d'Israélites s'efforçaient en effet " de se ravitailler auprès des cultivateurs dans des conditions illégales ", c'est-à-dire au «marché noir » qui prospérait et représentait un réel problème pour les autorités française et allemande qui cherchaient à l'endiguer. De nombreux Français procédaient de la sorte pour s'approvisionner à une période où les produits alimentaires étaient devenus de plus en plus rares chez les détaillants. La question de leur production et de leur distribution fut une autre constante dans les rapports mensuels envoyés au gouvernement par les préfets tout au long de la guerre. Le secrétaire général de la Police connaissait les difficultés du quotidien des Français aux prises avec les restrictions dès le début de l'Occupation.

Autre sujet de mécontentement : l'instauration du Service du Travail Obligatoire (STO), par la loi du 16 février 1943 en remplacement du système dit de Relève, avait dressé une partie de l'opinion et des jeunes générations contre l'occupant et le régime de Vichy. Ce rejet avait créé des solidarités nouvelles entre tous les illégaux et nourri un vivier pour la résistance. Ainsi le deuxième argument produit dans la circulaire vise les Juifs-Israélites qui auraient l'outrecuidance de se livrer à « une propagande anti-gouvernementale ». Et, de plus, ils étaient et restaient nombreux selon le décompte des forces d'occupation, ce qui justifiait un nécessaire renforcement de leur surveillance. Les pouvoirs publics manifestaient leur 
inquiétude face à ces Français qui adhéraient de moins en moins aux valeurs de la France nouvelle.

Bousquet avait également mesuré l'impact des rafles sur l'opinion publique, dans les villes et dans les campagnes. Pendant l'été 1942, les opérations policières menées sans ménagement et souvent avec brutalité, les arrestations de familles, d'enfants, de vieillards, de malades, avaient ému et même alerté les Français. Dans le même temps, les prises de position de certains hommes éminents de l'Église, la politique des otages avaient progressivement concouru au revirement de l'opinion. Bousquet craignait que la construction du discours antisémite de stigmatisation, de rejet ne soit plus suffisant en 1943 pour atteindre les objectifs de la politique nazie de déjudaïsation. L'un des recours possible pour lui était de cibler les ruraux et le mécontentement de la population, des Français et de le diriger encore une fois contre les Juifs.

\section{Bousquet face aux préfets}

Cette circulaire où l'on peut deviner une critique implicite de l'action des préfets, a permis à Bousquet de leur signifier que les forces de police n'avaient pas fait leur travail ou, à tout le moins, avaient relâché l'attention, diminué les contrôles. Or, la question du maintien de l'ordre qui était la tache par excellence des préfets, ne cessait de croître en importance, à mesure de l'évolution de la guerre et de la montée en puissance de la Résistance. Bousquet est venu alors en renfort de l'occupant en intervenant auprès des fonctionnaires chargés de l'ordre, de l'application des lois antisémites et des arrestations : il fit pression sur les préfets en demandant à chacun d'entre eux de s'impliquer «personnellement» dans la mise en place effective des instructions ministérielles. L'emploi de cet adverbe était un rappel clair et sans équivoque au serment que les préfets et hauts fonctionnaires avaient prêté le 19 février 1942 dans le grand salon de l'hôtel de ville de Vichy devant le maréchal Pétain. C'est d'ailleurs le préfet Fernand Carles qui avait prononcé au nom des 114 fonctionnaires réunis la formule de serment: « Je jure fidélité à la personne du chef de l'État et je m'engage à exercer ma charge pour le bien de l'État selon les lois de l'honneur et de la probité », comme on l'entend dans un film d'actualités de l'époque ${ }^{11}$.

${ }^{11}$ AD59 1W43, Cérémonie de la prestation de serment. https://www.ina.fr/video/AFE85000748 : Le serment des préfets au maréchal Pétain, $32^{\prime}$. 
Les fonctionnaires relevant du secrétariat d'État à l'Intérieur avaient dû également se soumettre à cette même prestation de serment en application de la loi du 10 avril $1942^{12}$. Cette volonté de rappeler les liens personnels et institutionnels qui unissaient les fonctionnaires à un État autoritaire, dont le chef était l'incarnation, pendant l'année cruciale que fut 1942, avait pourtant montré toutes ses limites. Même si la résistance policière doit être relativisée, la hiérarchie était consciente au fur et à mesure de l'évolution du conflit que les gendarmes, davantage présents dans le monde rural, et les policiers travaillaient parfois à contrecœur, montraient peu de zèle et d'initiative ou parfois même tentaient de saboter l'accomplissement de certaines taches lors des rafles ou dans la recherche des réfractaires du STO.

Une fois dressé l'état des lieux qu'il nommait « cet état de choses », Bousquet entendit passer à l'action, à la riposte auprès des préfets en utilisant le cadre administratif traditionnel et le maillage du territoire français: il appartenait désormais à chaque préfet de région et de département réduit à un rôle d'exécutant, à tous les services de police, de mettre en place «une surveillance constante [des] israélites». Cela impliquait un contrôle renforcé et sans relâche de tous les Juifs déjà recensés. Les autorités devaient débusquer les clandestins, ruraux ou citadins, les contrevenants à la loi générale et à la réglementation. C'est ainsi que Léon Karmiol, commerçant en lingerie à Lille rue du Court Debout, et sa femme, qui ne pouvaient plus travailler et dont les ressources s'épuisaient, étaient aux abois comme bon nombre de Juifs. Ils furent arrêtés le 4 avril 1944 au café «Double Bock» à Wazemmes dans le quartier populaire de Lille, alors que le mari cherchait à négocier une " chevalière en or avec brillant ${ }^{13}$. Ils avaient été dénoncés par Marcelle Bru-Boon, agent indicateur au service de Joseph Robyns.

Cette circulaire avait également pour but d'actualiser les listes de recensement des Juifs-Israélites déjà fort nombreuses. Sans que cela n'apparaisse dans ce texte officiel, il est évident que la police avait recours aux indicateurs et aux dénonciations.

Ainsi, " tout Israélite » qu'il soit étranger, citoyen français, dénaturalisé, était à la merci des autorités allemandes et françaises dès lors

\footnotetext{
${ }^{12}$ AD59 1W935, Application de la loi du 10 avril 1942 sur la prestation de serment de fidélité des fonctionnaires.

${ }^{13}$ AD59 9W824, Procès de Germain Bru et de Marcelle Bru née Boon et AD59 9W41, Procès de Joseph Robyns. Ils ont été condamnés pour intelligence avec l'ennemi et pour avoir livré des Israélites aux Allemands. Et témoignage de Suzanne Meller, née Karmiol, le 20 janvier 2020.
} 
qu'il était en infraction et quelle qu'en soit la gravité. S'il est question de mesure d'internement, l'arrestation et la déportation étaient souvent le lot des contrevenants. Beaucoup, y compris des Français de confession juive et devant porter l'étoile juive, furent déportés par mesures de représailles.

Le document se termine abruptement par une injonction et surtout le devoir « de donner immédiatement des ordres fermes » pour l'application de cette circulaire.

$* * * * * * * * * *$

Ce texte a été conçu avec le plus grand des cynismes, a repris en les énonçant de façon subtile les poncifs de l'antisémitisme. Il était destiné à manipuler l'opinion pour tenter de la retourner par l'intermédiaire du corps préfectoral et des services de police. Son application et son objectif ultime ne visaient qu'à rendre les Juifs encore plus vulnérables, les arrêter et les « envoyer à l'est ». La traque des Juifs dont le nombre de « déportables » se réduisait, était de plus en plus manifeste à partir de l'été 1943. 